\section{Darstellung und Kristallstruktur von CaLiSb}

(Ternäre E-Phasen von Hauptgruppenelementen II)

\section{B. Eisenmann, O. Liebrich, Herbert Schäfer und ARMIN WeISS}

Institut für Anorganische Chemie der Universität München

(Z. Naturforschg. 24 b, 1344-1345 [1969]; eingegangen am 29. Juli 1969)

Intermetallische Verbindungen der Elemente der 4. und 5. Hauptgruppe mit Übergangselementen bilden bei geeigneter Stöchiometrie $\left(\mathrm{A}_{2} \mathrm{X}\right.$ bei binären, $\mathrm{ABX}$ bei ternären Phasen mit $\mathrm{A}, \mathrm{B}=$ Utbergangselement, $\mathrm{X}$ $=$ Element der IV. oder V. Hauptgruppe) und günstigen Größenverhältnissen häufig das Anti-Blei(II) chlorid-Gitter aus ${ }^{1}$. Ternäre Phasen dieses Typs mit geordneter Verteilung der A,B-Atome werden als E-Phasen bezeichnet ${ }^{3}$. Wie wir vor kurzem zeigen konnten, kann dieser Typ auch dann ausgebildet werden, wenn A und B Hauptgruppenelemente sind, wie z. B. in $\mathrm{CaMgSi}, \mathrm{CaMgGe}$ und $\mathrm{CaMgSn}^{2}$. Als neuer Vertreter dieses Bautyps wurde nun das $\mathrm{CaLiSb}$ gefunden.

Zur Darstellung von CaLiSb wurden stöchiometrische Mengen der Elemente in einem Eisentiegel unter Argonatmosphäre bei $980{ }^{\circ} \mathrm{C}$ zusammengeschmolzen und $40 \mathrm{Stdn}$. bei $600{ }^{\circ} \mathrm{C}$ getempert. Dabei erhielten wir einen nadelig kristallisierenden, metallischen, dunkelgrauen Regulus, der an feuchter Luft unter Abscheidung schwarzer Zersetzungsprodukte zerfiel. Die Analyse ausgelesener Einkristallkonglomerate ergab ein Verhältnis $\mathrm{Ca}: \mathrm{Li}: \mathrm{Sb}$ von $1: 1: 1$.

W e i $ß$ e n b e r g-Aufnahmen zeigten, daß die Verbindung den bereits beschriebenen $\mathrm{CaMgSi}, \mathrm{CaMgGe}$ und $\mathrm{CaMgSn}$ isotyp ist. Die kristallographischen Daten sind in der Tab. 1 zusammengestellt.

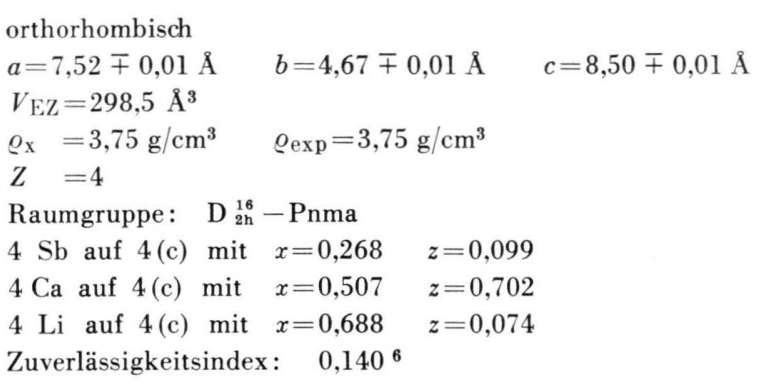

Tab. 1. Kristallographische Daten von CaLiSb.

Die genaue Parameterbestimmung zeigte erneut ${ }^{4}$ die bemerkenswerte Flexibilität des Gitters; durch relativ geringe Parameteränderungen werden recht verschie-

1 F. X. Spiegel, D. Bardos u. P. A. Beck, Trans. metallurg. Soc. AIME 227, 575 [1963]; C. B. Shoemaker u. D. P. Shoemaker, Acta crystallogr. [Copenhagen] 18, 900 [1965]; E. Gangelberger, H. Nowotny u. F. Benesovsky, Mh. Chem. 98, 95 [1967] ; J. Nickl u. H. SPREnger, Naturwissenschaften 54, 18 [1967] ; S. RUNDQVIST u. P. TANSURIwongs, Acta chem. scand. 21, 813 [1967] ; S. RundQvist u. P. NAwAPONG, 20, 2250 [1966]. dene Koordinationszahlen möglich. Verglichen mit $\mathrm{CaMgSn}^{2}$ nehmen die Sb-Atome im CaLiSb die Zinnplätze ein. Der Koordinationspolyeder ist aber aufgeweitet; neben acht nächsten Nachbarn müssen zwei weitere, etwas entfernter liegende Atome berücksichtigt werden. Die Calciumatome, die in CaMgSn 15 Nachbarn haben, können im CaLiSb $15+1$ Nachbarn um sich gruppieren. Dies wird offensichtlich durch das kleinere Lithiumatom bedingt. Die Lage der Lithiumatome ist wegen des geringen Streubeitrags allerdings nur ungenau zu bestimmen. Die von seinen Legierungspartnern frei gelassene Lücke wie auch der bei der Dif-

\begin{tabular}{|c|c|c|c|}
\hline & $\mathrm{Ca}$ & $\mathrm{Li}$ & $\mathrm{Sb}$ \\
\hline $\mathrm{Ca}$ & $\begin{array}{ll}3,78 & (2 \times) \\
4,16 & (2 \times)\end{array}$ & $\begin{array}{ll}3,44 & \\
3,36 & \\
3,45 & (2 \times) \\
3,35 & (2 \times)\end{array}$ & $\begin{array}{ll}3,23 & \\
3,34 & (2 \times) \\
3,24 & (2 \times)\end{array}$ \\
\hline $\mathrm{Li}$ & $\begin{array}{ll}3,36 & \\
3,44 & \\
3,35 & (2 \times) \\
3,45 & (2 \times)\end{array}$ & & $\begin{array}{ll}2,84 & \\
2,78 & (2 \times)\end{array}$ \\
\hline $\mathrm{Sb}$ & $\begin{array}{ll}3,23 & \\
3,34 & (2 \times) \\
3,24 & (2 \times)\end{array}$ & $\begin{array}{ll}2,84 & \\
2,78 & (2 \times)\end{array}$ & \\
\hline & Sb 3,83 & $\begin{array}{ll}\mathrm{Sb} & 3,16 \\
\mathrm{Li} & 3,88(2 \times)\end{array}$ & $\begin{array}{ll}\mathrm{Ca} & 3,83 \\
\mathrm{Li} & 3,16\end{array}$ \\
\hline
\end{tabular}

Tab. 2. Atomabstände in der Phase CaLiSb $[\AA]]$. (Unter der gestrichelten Linie sind Abstände aufgeführt, die deutlich länger sind als die kürzesten, aber doch noch so kurz. daß direkte Wechselwirkungen angenommen werden müssen.)

\begin{tabular}{|c|c|c|c|c|}
\hline \multirow{2}{*}{$\begin{array}{c}\text { Umgebung } \\
\text { der } \\
\text { Atome }\end{array}$} & \multicolumn{3}{|c|}{$\begin{array}{l}\text { Zahl und Art des } \\
\text { nächsten Nachbarn }\end{array}$} & \multirow[b]{2}{*}{$\begin{array}{c}\text { Summe de } \\
\text { nächsten } \\
\text { Nachbarn }\end{array}$} \\
\hline & $\mathrm{Ca}_{\mathrm{I}}$ & $\begin{array}{c}\mathrm{Mg} \\
\text { bzw. CaII } \\
\text { bzw. Li }\end{array}$ & $\begin{array}{c}\text { Sn } \\
\text { bzw. } \\
\text { Sb }\end{array}$ & \\
\hline \multicolumn{5}{|l|}{ im $\mathrm{Ca}_{2} \mathrm{Sn}$} \\
\hline $\mathrm{Ca}_{\mathrm{I}}$ & $0+4$ & 6 & 5 & $11+4$ \\
\hline CaII & 6 & 2 & $3+1$ & $11+1$ \\
\hline Sn & 5 & 4 & - & 9 \\
\hline \multicolumn{5}{|l|}{ im $\mathrm{CaMgSn}$} \\
\hline $\mathrm{Ca}$ & 4 & 6 & 5 & 15 \\
\hline $\mathrm{Mg}$ & 6 & 2 & 4 & 12 \\
\hline $\mathrm{Sn}$ & 5 & 4 & - & 9 \\
\hline \multicolumn{5}{|l|}{ im CaLiSb } \\
\hline $\mathrm{Ca}$ & 4 & 6 & $5+1$ & $15+1$ \\
\hline $\mathrm{Li}$ & 6 & $0+2$ & $3+1$ & $9+3$ \\
\hline $\mathrm{Sb}$ & $5+1$ & $3+1$ & - & $8+2$ \\
\hline
\end{tabular}

Tab. 3. Vergleich der Koordinationszahlen von $\mathrm{Ca}_{2} \mathrm{Sn}$, $\mathrm{CaMgSn}$ und CaLiSb.

2 H. Axel, B. Eisenmann, H. Schäfer u. A. Weiss, Z. Naturforschg., im Druck.

3 J. H. Westbrook, R. K. Dicerbo u. A. J. Peat, Gen. Electric Co GE-58 RL 2117 (1958).

4 W. Jeitschкo, Acta crystallogr. [Copenhagen] B 24, 930 [1968].

5 P. Eckerlin, E. Leicht u. E. Wölfel, Z. anorg. allg. Chem. 307, 145 [1961]. 
ferenz-Fourier-Synthese erscheinende Peak zeigen aber, daß für die Lithiumatome zunächst nur neun nächste Nachbarn diskutiert werden können. 3 weitere Nachbarn, die im CaMgSn um das dem Lithium entsprechende Magnesiumatom 12 nächste Nachbarn bedingen, sind deutlich weiter entfernt. In der Tab. 2 sind die Atomabstände zusammengefaßt.

In der Tab. 3 sind die Koordinationsverhältnisse in der Reihe $\mathrm{Ca}_{2} \mathrm{Sn}, \mathrm{CaMgSn}$ und $\mathrm{CaLiSb}$ miteinander verglichen. Im $\mathrm{Ca}_{2} \mathrm{Sn}$ überwiegt die Tendenz, eine gleichwertige Koordination um die metallischen Legierungspartner aufzubauen, entsprechend dem gleichen Radius. Die Packungsdichte erscheint allerdings nicht

6 Dem Leibniz-Rechenzentrum München danken wir für die Bereitstellung von Rechenzeit an der Telefunken TR4-Re- optimal, wenn man die recht unterschiedlichen Atomabstände beachtet ${ }^{5}$. Offensichtlich kommen die Radienverhältnisse der ternären Verbindung CaMgSn der Atompackung dieses Strukturtyps besser entgegen. Die Atomabstände sind hier sehr gleichmäßig und erklären den zunächst merkwürdigen Befund, daß beim Ersatz des Calciums durch das leichtere Magnesium die Dichte erhöht wird ${ }^{2}$. Im CaLiSb nähert sich der Radius des einen metallischen Legierungspartners ( $=$ Lithium) dem Radius des Halbmetalls (= Antimon) an. Der Gitterverband trägt diesem Wechsel Rechnung, indem für das Lithium die dem Halbmetall entsprechende Koordination angestrebt wird.

chenanlage. Der Deutschen Forschungsgemeinschaft danken wir für die Unterstützung dieser Arbeit.

\section{Die Kristallstruktur des Ammoniumtetrathio- antimonats $\left(\mathrm{NH}_{4}\right)_{3} \mathrm{SbS}_{4}$}

\section{H. Graf, Herbert Schäfer und Armin Weiss}

Institut für Anorganische Chemie der Universität München

(Z. Naturforschg. 24 b, 1345-1346 [1969] ; eingegangen am 29. Juli 1969)

Im Gegensatz zu Ammoniumtetrathioarsenat $\left(\mathrm{NH}_{4}\right)_{3} \mathrm{AsS}_{4}{ }^{1}$, das mit $\left(\mathrm{NH}_{4}\right)_{3} \mathrm{VS}_{4}$ und $\mathrm{K}_{3} \mathrm{PS}_{4}$ isotyp ist ${ }^{2,3}$, bildet das bisher in der Literatur noch nicht beschriebene, wasserfreie Ammoniumtetrathioantimonat $\left(\mathrm{NH}_{4}\right)_{3} \mathrm{SbS}_{4}$ einen eigenen Strukturtyp aus. Es kristallisiert in farblosen Rhombendodekaedern, die sich an feuchter Luft unter $\mathrm{H}_{2} \mathrm{~S}$ - und $\mathrm{NH}_{3}$-Abspaltung sofort mit einer rotbraunen Kruste überziehen.

Die Auswertung von Einkristallaufnahmen nach dem WeIsSENBERG-Verfahren ergab eine kubisch innenzentrierte Elementarzelle, die zwei Formeleinheiten enthält. Die gesetzmäßigen Interferenzbedingungen lassen die beiden Raumgruppen $\mathrm{I} 23-\mathrm{T}^{3}$ und $\mathrm{I} 43 \mathrm{~m}-\mathrm{T}_{\mathrm{d}}^{3}$ zur Auswahl (Tab.1). Die Struktur wurde in der

\begin{tabular}{lc}
\hline kubisch, & $a=7,940 \pm 0,005 \AA$ \\
$V_{\mathrm{EZ}}=500,6 \AA^{3}$ & $z=2$, \\
$d_{\mathrm{x}}=2,02 \mathrm{~g} / \mathrm{cm}^{3}$ & $d_{\exp }=2,05 \mathrm{~g} / \mathrm{cm}^{3}$ \\
Raumgruppe: $\mathrm{I} 43 \mathrm{~m} \cdot-\mathrm{T}_{\mathrm{d}}^{3}$ & \\
$2 \mathrm{Sb}$ in 2(a) & \\
$6 \mathrm{NH}_{4}$ in 6(b) & \\
$8 \mathrm{~S}$ in 8(c) mit & $x=0,171$ \\
Zuverlässigkeitsindex : & $0,114^{6}$ \\
\hline
\end{tabular}

Tab. 1. Kristallographische Daten des $\left(\mathrm{NH}_{4}\right)_{3} \mathrm{SbS}_{4}$.

höhersymmetrischen beschrieben. Die Antimonatome sitzen in den Ecken und im Mittelpunkt des Elementarwürfels, während die Ammoniumionen die Mittel-

1 H. Schäfer, G. Schäfer u. A. Weiss, Z. Naturforschg. 18 b, 665 [1963].

2 H. SChäfer, P. Moritz u. A. Weiss, Z. Naturforschg. 20 b, 603 [1965]. punkte der Würfelflächen und -kanten einnehmen. Jeweils vier Schwefelatome bilden um die Antimonatome einen regulären Tetraeder aus. Die $\mathrm{Sb}-\mathrm{S}$-Abstände betragen $2,35 \AA$, die $\mathrm{S}-\mathrm{S}$-Abstände innerhalb eines Tetraeders ergeben sich zu $3,84 \AA$, die kürzesten $\mathrm{S}-\mathrm{S}$ Abstände zwischen zwei Tetraedern sind 4,35 $\AA$. Im ebenfalls kubisch kristallisierenden $\mathrm{S}$ chlip pe schen Salz $\mathrm{Na}_{3} \mathrm{SbS}_{4} \cdot 9 \mathrm{H}_{2} \mathrm{O}$ wurde ein $\mathrm{Sb}-\mathrm{S}$-Abstand von $2,37 \AA$ gefunden ${ }^{4}$.

Jedes Ammoniumion ist von acht Schwefelatomen umgeben (Abb. 1). Jeweils vier dieser Schwefelnachbarn sind bezüglich der Ammoniumlage gleichwertig und bilden für sich einen verzerrten Tetraeder um diese Lage aus. In einem Fall errechnet man einen $\mathrm{NH}_{4}-\mathrm{S}$ Abstand von $3,24 \AA$ (A), im anderen Fall 3,94 $\AA$ (B). Diese beiden Tetraeder, die das Ammoniumion als gemeinsamen Schwerpunkt haben, bilden zusammen ein Koordinationspolyeder, das man als verzerrtes trigonales Dodekaeder beschreiben kann (Abb. 1). Je drei Schwefelatome bilden gleichschenklige Dreiecke, die sich nur in der Basis unterscheiden. Bei acht Dreiecken beträgt die Basis $5,89 \AA$ (c), bei den vier weiteren Dreiecken nur $3,84 \AA$ (a) (=S-S-Abstand innerhalb eines $\mathrm{SbS}_{4}$-Tetraeders). Die Schenkel aller zwölf Dreiecke sind 4,35 $\AA$ (b) lang und entsprechen dem kürzesten $\mathrm{S}-\mathrm{S}$-Abstand direkt benachbarter $\mathrm{SbS}_{4}$-Tetraeder.

Die Struktur des $\left(\mathrm{NH}_{4}\right)_{3} \mathrm{SbS}_{4}$ hat Ähnlichkeit mit der von $\mathrm{Ag}_{3} \mathrm{PO}_{4}$ bzw. $\mathrm{Ag}_{3} \mathrm{AsO}_{4}{ }^{5}$, die ebenfalls kubisch kristallisieren. Die Anordnung der Anionen $\mathrm{BX}_{4}{ }^{3} \ominus$ $\left(\mathrm{PO}_{4}{ }^{3 \ominus}, \mathrm{AsO}_{4}{ }^{3 \ominus}\right.$ bzw. $\left.\mathrm{SbS}_{4}{ }^{3}{ }^{\ominus}\right)$ ist in beiden Strukturen gleich. Bei den Sauerstoffverbindungen werden die kleinen Kationen allerdings in Tetraederlücken eingebaut, während die wesentlich größeren Ammoniumionen in der Schwefelverbindung die beschriebenen Dodekaederlücken einnehmen.

Zur Darstellung des wasserfreien $\left(\mathrm{NH}_{4}\right)_{3} \mathrm{SbS}_{4}$ wurden in $70 \mathrm{ml}$ einer konzentrierten wäßrigen Lösung von

${ }^{3}$ H. Schäfer, G. Schäfer u. A. Weiss, Z. Naturforschg. 20 b. 811 [1965].

4 A. Grund u. A. Preisinger, Acta crystallogr. [Copenhagen] 3, 363 [1950]. 